\title{
Temperature fields induced by geothermal devices
}

\author{
V. Ciriello ${ }^{\mathrm{a}}$, M. Bottarelli ${ }^{\mathrm{b}}$, V. Di Federico ${ }^{\mathrm{a}}$, D. M. Tartakovsky ${ }^{\mathrm{c}, *}$ \\ ${ }^{a}$ Department of Civil, Chemical, Environmental, and Materials Engineering, University of \\ Bologna (Italy) \\ ${ }^{b}$ Department of Architecture, University of Ferrara, Ferrara, Italy. \\ ${ }^{c}$ Department of Mechanical and Aerospace Engineering, University of California, San Diego \\ (USA)
}

\begin{abstract}
Efficient and sustainable exploitation of low-enthalpy geothermal energy relies on accurate representations of heat transfer processes in the subsurface. An analytical model, which provides such a representation by predicting the dynamics of thermal fields induced by shallow ground heat exchangers (GHEs), is derived. The model accounts for atmospheric temperature fluctuations at the ground surface, an arbitrary geometry of GHEs operating in time-varying heating/cooling modes, and anisotropy and uncertain spatio-temporal variability of thermal conductivity of the ambient soil. To validate the model, its predictions of a thermal fields generated by a shallow flat-panel GHEs are compared with experimental data. This comparison demonstrates the model's ability to provide accurate fit-free predictions of soil-temperature fields generated by GHEs. The analysis presented shows that a single horizontal GHE may affect soil temperature by several degrees at distances on the order of one meter. The volume of influence is expressed in terms of soil thermal properties. Such modeling predictions are invaluable for screening of potential sites and optimal design of geothermal systems consisting of multiple GHEs.
\end{abstract}

Keywords: temperature, subsurface, modeling, heat exchangers

\footnotetext{
* Corresponding author

Email address: dmt@ucsd.edu (D. M. Tartakovsky)
}

Preprint submitted to Energy

August 26, 2015

(C) 2015. This manuscript version is made available under the Elsevier user license http://www.elsevier.com/open-access/userlicense/1.0/ 


\section{Introduction}

Atmospheric temperature fluctuations affect soil temperature at depths of up to $20 \mathrm{~m}$ below the ground surface. Thermal inertia of this subsurface region induces both attenuation and time delay of surface temperature. As a 5 result, temperature of the subsurface is higher/lower than that of air during the cold/hot seasons. (At depths exceeding $20 \mathrm{~m}$, subsurface temperature is not affected by its atmospheric counterpart; it is controlled, instead, by the geothermal gradient.) Ground heat exchangers (GHEs) exploit such differences between air and soil temperatures for heating/cooling purposes [1. Among them, ground-coupled heat pump systems are regarded as a sustainable and cost-effective technology 2. These systems couple a heat pump with the ground via a closed loop through which a working fluid circulates; the heat exchange with the ground occurs by means of GHEs (Figure 1), which are located either vertically or horizontally at various depths 3 . Horizontal GHEs typically provide little energy, but are cheaper, more compliant with the environment, and easier to operate and maintain. In this configuration, the ground mainly serves as a solar energy buffer [e.g., 4, 5, 6].

Success of any GHE ultimately depends upon the ambient soil temperature field (STF) it generates. The latter is used as a key metric in designing GHEs and assessing their effect on the subsurface environment. For example, a GHE used in the heating of a building might cool the ambient soil to the point at which either the GHE operation becomes uneconomical or subsurface biological processes become unsustainable. A shallow horizontal GHE can change soil temperature by several degrees Celsius, with appreciable changes confined to

25 its neighborhood of radius on the order of one meter [7, 8. The efficiency of such devices rests on one's ability to optimize the surface available for heat transfer and to reduce the mutual interference between exchangers. The former venue was pursued by exploring various GHE geometries, including slinky coils, radiators, and spirals [e.g., 9, and the references therein], as well as flat panels [7]. 30 A flat-panel GHE affects larger volumes of the ambient soil than a radiator GHE 

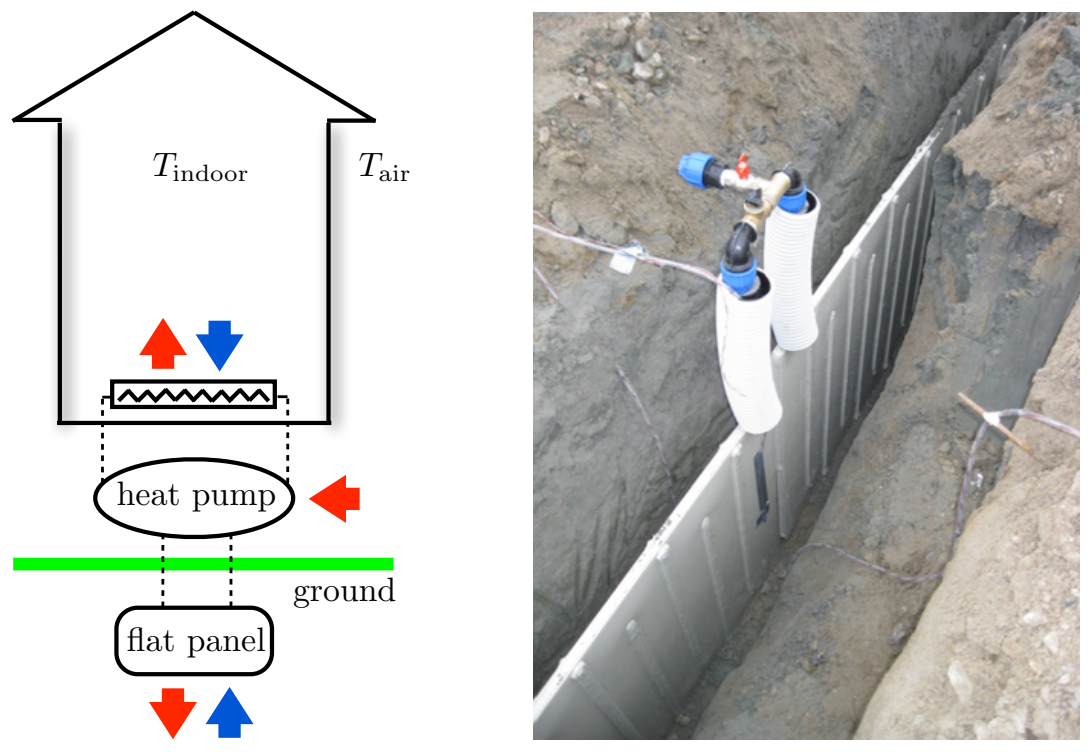

Figure 1: Left: Schematic representation of a ground-coupled heat pump system. Right: shallow ground heat exchanger with a flat-panel geometry.

does, reducing soil-temperature oscillations. For the same surface of exchange, a flat-panel GHE has lower thermal resistance, resulting in higher efficiency [7].

Regardless of the technology employed, quantification of energy that can be either retrieved or stored in the subsurface requires an accurate estimation of the ambient STF. This task is challenging due to STF's sensitivity to atmospheric dynamics, soil heterogeneity, and spatio-temporal variability of soil water content [10, 11, 12, 13, 14. Although it has been argued that soil heterogeneity might play a minor role in the overall performance of shallow GHEs [15, the impact of temporal variability of soil water content (e.g., due to infiltration and/or 40 evaporation) on the soil's thermal properties and, hence, on the GHE performance is undeniable. Models that treat soil properties as constants have been shown to yield inaccurate predictions of STFs, especially in shallow soils, [e.g., 16, 11, 13. The minimal model complexity that is necessary to describe the STF dynamics is another potential source of error. While many studies [e.g., 17, 18] 45 rely on one-dimensional heat conduction equations to estimate vertical soil tem- 
perature profiles under natural conditions, the presence of GHEs increases the modeling complexity.

Here, a general mathematical framework is presented to analytically predict the dynamics of the STFs induced by GHEs in ambient shallow soils. This 50 framework accounts for atmospheric temperature fluctuations at the ground surface, an arbitrary shape and number of GHEs, anisotropy of soil thermal properties, and their spatial variability and spatio-temporal dependence on soil water content. Temporal fluctuations of both surface temperature and soil thermal diffusivity are handled exactly; uncertainty due to spatial variability of soil thermal diffusivity is tackled by employing the effective medium theory [e.g., [19, and the references therein]. This formulation significantly extends the range of predictive analytical models available in the field. It can be employed for a screening-level assessment of potential geothermal sites and for verification of numerical codes.

Section 2 provides a model formulation, including modeling assumptions. A general three-dimensional analytical solution of this problem and its (twodimensional) application to horizontal flat-panel GHEs are presented in Section 3. In Section 4, the model is validated by comparing its predictions of the STF with the experimental data collected at a field in the vicinity of Ferrara,

65 Italy. Section 5 demonstrates the model's utility by forecasting the STF dynamics induced by operation of a single GHE used to meet the energy requirement of a building during the cold season. A summary of the key findings is provided in Section 6 .

\section{Model Formulation}

The subsurface is treated as a semi-infinite domain, $\Omega=\left\{\mathbf{x}=\left(x_{1}, x_{2}, x_{3}\right)^{\top}\right.$ : $\left.-\infty<x_{1}, x_{2}<\infty, 0 \leq x_{3}<\infty\right\}$, and a (possibly multi-connected) region occupied by a GHE is denoted by E. A macroscopic (Darcy-scale) description of subsurface temperature, $T(\mathbf{x}, t)$, at any "point" $\mathbf{x}$ and time $t$ is provided by 
a heat conduction equation

$$
\rho c \frac{\partial T}{\partial t}=\nabla \cdot(\mathbf{K} \nabla T)+g, \quad t>0, \quad \mathbf{x} \in \Omega,
$$

70 of the soil, respectively; and $g(\mathbf{x}, t)$ represents the heat source generated by the GHE, such that $g(\mathbf{x}, t) \equiv 0$ for $\mathbf{x} \notin E$. The three soil parameters, $\rho, c$ and $\mathbf{K}$, vary, to different degrees, in space and time due to soil heterogeneity and changing water content [e.g., 13, and the references therein]. Soil anisotropy are aligned with the coordinate system, such that the off-diagonal components of this tensor are $K_{i j}=0$ for $i \neq j$. Without loss of generality, we set $K_{11}=$ $K_{22}=K_{h}$ and $K_{33}=K_{v}$, where $K_{h}$ and $K_{v}$ are the horizontal and vertical thermal conductivities, respectively.

Equation (1) is subject to an initial condition

$$
T(\mathbf{x}, 0)=T_{m},
$$

where $T_{m}$ is the average temperature of soil in the stable layer; it is commonly set to the average temperature of air [4, 20]. This temperature varies in response to atmospheric fluctuations at the ground surface $\left(x_{3}=0\right)$, which manifest themselves through a boundary condition

$$
T\left(x_{1}, x_{2}, 0, t\right)=T_{m}-A \cos (\omega t) .
$$

Here $\omega=2 \pi / 365$ is the fluctuation frequency, with time $t$ expressed in Julian days; and $A$ denotes the yearly amplitude of thermal oscillations at the ground surface. Ground temperature fluctuations do not affect the soil temperature far below the ground surface, which gives rise to a boundary condition

$$
T\left(x_{1}, x_{2}, \infty, t\right)=T_{m} .
$$

To ensure that the temperature response to the localized sources remains finite, a boundary condition

$$
|T(\mathbf{x}, t)|<\infty, \quad x_{1}^{2}+x_{2}^{2} \rightarrow \infty
$$

80 is imposed. 


\subsection{Modeling Assumptions}

The following assumptions facilitate the subsequent derivation of analytical solutions of the boundary-value problem (1)-(3).

1. Soil heterogeneity and spatial variation of water content affect primarily the soil's thermal conductivity; all the parameters change with time, $\rho=$ $\rho(t), c=c(t)$ and $\mathbf{K}=\mathbf{K}(\mathbf{x}, t)$.

2. Available measurements of thermal diffusivity tensor $\boldsymbol{\kappa} \equiv \mathbf{K} /(\rho c)$ are sufficient to reliably estimate its sample statistics (mean, variance, and correlation function); the ergodicity hypothesis holds, allowing one to interchange these spatial statistics for their ensemble counterparts.

3. Spatial variability of thermal diffusivity tensor $\kappa$ is small, such that the variance of the logarithm of its largest component, $k=\ln \left(\max \left\{\kappa_{h}, \kappa_{v}\right\}\right)$, is $\sigma_{k}^{2}<1$.

4. Thermal gradients, $|\nabla T|$, are sufficiently smooth in the mean (vary slowly in space and time) to allow for localization of the otherwise nonlocal (integro-differential) equations governing the (ensemble) average dynamics of the $\operatorname{STF}\langle T(\mathbf{x}, t)\rangle$.

\subsection{Problem Transformation}

Assumption 1 enables one to rewrite (1) in terms of the thermal diffusivity tensor $\boldsymbol{\kappa}(\mathbf{x}, t)$,

$$
\frac{\partial T}{\partial t}=\nabla \cdot(\kappa \nabla T)+f, \quad t>0, \quad \mathbf{x} \in \Omega
$$

where $f(\mathbf{x}, t) \equiv g /(\rho c)$.

Heterogeneity of the subsurface environment, combined with limited information about spatio-temporal variability water content and, hence, the thermal diffusivity $\boldsymbol{\kappa}$, renders the latter uncertain. This uncertainty is commonly quantified by treating such input parameters as random fields, whose ensemble statistics are inferred from spatial data (Assumption 2) [e.g., 21, and the references therein]. Given uncertainty in $\kappa$, the best estimate of the STF is given 
by the ensemble mean temperature $\langle T(\mathbf{x}, t)\rangle$. Under Assumptions 3 and 4 , the latter satisfies an equation

$$
\frac{\partial\langle T\rangle}{\partial t}=\nabla \cdot\left(\boldsymbol{\kappa}_{\mathrm{eff}} \nabla\langle T\rangle\right)+f, \quad t>0, \quad \mathbf{x} \in \Omega
$$

which is obtained by stochastic averaging of (4) [19, 22. The time-dependent effective thermal conductivity tensor, $\boldsymbol{\kappa}_{\text {eff }}(t)$, is expressed in terms of the known statistics of $\boldsymbol{\kappa}(\mathbf{x}, t)$, i.e., its mean, variance, and correlation function (see Equations 13 and 14 in [19]).

The effective thermal conductivity tensor is expressed as the product $\boldsymbol{\kappa}_{\text {eff }}(t)=$ $\alpha(t) \boldsymbol{\kappa}^{\mathrm{e}}$, where the dimensionless function $\alpha(t)$ quantifies the temporal variability of the effective (averaged) thermal conductivity due to spatially averaged changes in water content; and $\boldsymbol{\kappa}^{\mathrm{e}}$ is the instantaneous thermal conductivity tensor. The off-diagonal components of this tensor are $\kappa_{i j}^{\mathrm{e}}=0$ for $i \neq j$, and $\kappa_{11}^{\mathrm{e}}=\kappa_{22}^{\mathrm{e}}=\kappa_{h}^{\mathrm{e}}$ and $\kappa_{33}=\kappa_{v}^{\mathrm{e}}$, where $\kappa_{h}^{\mathrm{e}}$ and $\kappa_{v}^{\mathrm{e}}$ are the instantaneous average horizontal and vertical thermal diffusivity, respectively. The term "instantaneous" refers to the values of $\kappa_{h}^{\mathrm{e}}$ and $\kappa_{v}^{\mathrm{e}}$ for a given value of water content. Then the change of coordinates

$$
\tilde{x}_{1}=x_{1} \sqrt{\frac{\tilde{\kappa}}{\kappa_{h}^{\mathrm{e}}}}, \quad \tilde{x}_{2}=x_{2} \sqrt{\frac{\tilde{\kappa}}{\kappa_{h}^{\mathrm{e}}}} \quad \text { and } \quad \tilde{x}_{3}=x_{3} \sqrt{\frac{\tilde{\kappa}}{\kappa_{v}^{\mathrm{e}}}}
$$

transforms (5) into

$$
\frac{\partial\langle T\rangle}{\partial t}=\alpha(t) \tilde{\kappa} \tilde{\nabla}^{2}\langle T\rangle+f, \quad t>0, \quad \tilde{\mathbf{x}} \in \Omega
$$

where $\tilde{\kappa}=\sqrt{\kappa_{h}^{\mathrm{e}} \kappa_{v}^{\mathrm{e}}}$ is the geometric mean of the thermal diffusivity, $\tilde{\nabla}^{2}$ denotes the Laplacian operator in the $\tilde{\mathbf{x}}$ coordinate system, and $f(\tilde{\mathbf{x}}, t)$ vanishes outside the transformed domain $\tilde{E}$ occupied by the GHE. Finally, a new time variable,

$$
\tilde{t}=\int_{0}^{t} \alpha(\tau) \mathrm{d} \tau,
$$

is introduced to transform (7) into a heat conduction equation with constant thermal diffusivity $\tilde{\kappa}$,

$$
\frac{\partial\langle T\rangle}{\partial \tilde{t}}=\tilde{\kappa} \tilde{\nabla}^{2}\langle T\rangle+\tilde{f}(\tilde{\mathbf{x}}, \tilde{t}), \quad \tilde{t}>0, \quad \tilde{\mathbf{x}} \in \Omega,
$$


where $\tilde{f}(\tilde{\mathbf{x}}, \tilde{t}) \equiv f(\tilde{\mathbf{x}}, t) / \alpha(t)$.

To sum up, equation (99), subject to the transformed initial and boundary conditions, provides the best estimate of the STF induced by a GHE operating in a soil with uncertain thermal diffusivity. If the thermal diffusivity is spatially uniform and known with certainty, this boundary-value problem describes a unique STF, rather then its estimate, i.e., $T \equiv\langle T\rangle$. Also, no transformation of coordinates is necessary if the medium is isotropic and the thermal diffusivity does not change with time. In the following, we drop the tilde and $\langle\cdot\rangle$ to simplify the notation.

\subsection{Example of Flat-Panel GHEs}

A GHE composed of $N$ identical flat panels of height $h$ provides an example of the otherwise general source term $f(\mathbf{x}, t)$ and GHE shape $E$. The panels are buried at depth $x_{3}=d$ at locations $\left(x_{1}=\xi_{i}, x_{2}=\zeta_{i}\right)$, where $i=1 \ldots, N$. In a typical GHE, the flat panels are elongated and combined in a series to form a line. This allows one to adopt a two-dimensional representation of the heat exchange between the devices and the surrounding soil. This fact is emphasized by using the notation $x=x_{1}$ and $z=x_{3}$ (Figure 2).
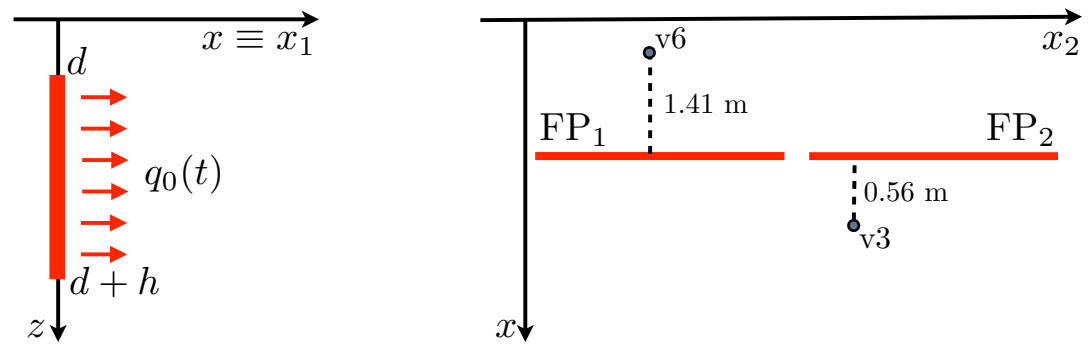

Figure 2: Vertical (left) and horizontal (right) cross-sections of the subsurface with a GHE composed of flat panels. The horizontal cross-section exhibits two flat panels and two sensors, v3 and v6, located at depths $z=2.03$ and $2.57 \mathrm{~m}$, respectively. Soil-temperature measurements provided by these sensors are used for model validation.

Let $q_{0}(t)$ denote the heat flux (heat power per unit surface) prescribed on each panel of the GHE, which operates during a time interval $\left[t_{0}, t_{1}\right]$. Then, the 
source term in $(9)$ takes the form

$$
f(x, z, t)=\frac{\kappa}{K} \mathcal{I}(x, z) q_{0}(t) \mathcal{H}\left(t-t_{0}\right) \mathcal{H}\left(t_{1}-t\right),
$$

where the indicator (or membership) function $\mathcal{I}(x, z)$ is defined by

$$
\mathcal{I}(x, z)=\mathcal{H}(z-d) \mathcal{H}(d+h-z) \sum_{i=1}^{N} \delta\left(x-\xi_{i}\right)
$$

and $\delta(\cdot)$ and $\mathcal{H}(\cdot)$ are the Dirac delta and Heaviside functions, respectively. The time-dependence of the heat flux $q_{0}$ accounts for operating conditions, in which GHEs may start and stop working several times per hour and the operating period may vary significantly from day to day. This happens because the heat flux is controlled by a ground-coupled heat pump, which operates in order to assure a target indoor temperature of building. In this sense, the heat flux is linked to the temperature fluctuations at the ground surface [20]. This behavior is represented by considering the operation of GHEs to be piece-wise constant during each day, so that $t_{0}$ and $t_{1}$ are expressed in Julian days. A different value of heat flux is assigned to each day, given by the ratio between the total energy exchanged during that day and the effective operating period of the GHE. This captures the system's behavior at the daily scale.

\section{Analytical Solutions}

Green's functions are used to derive a general three-dimensional solution

of (9) with an arbitrary source (GHE shape) and its two-dimensional counterpart corresponding to $f$ in 10 .

\subsection{Arbitrarily Shaped GHE}

A closed-form analytical expression for Green's function $G\left(\mathbf{x}, \mathbf{x}^{\prime}, t-t^{\prime}\right)$ for the boundary-value problem (9), (2) and (3) is given by (A.2)-A.4 of Appendix A. The STF $T(\mathbf{x}, t)$ induced by a GHE of an arbitrary shape $E$ is computed as $T=T_{m}-\kappa A \int_{0}^{t} \cos \left(\omega t^{\prime}\right) \int_{-\infty}^{+\infty} \frac{\partial G}{\partial x_{3}^{\prime}}\left(\cdot, x_{3}^{\prime}=0, \cdot\right) \mathrm{d} x_{1}^{\prime} \mathrm{d} x_{2}^{\prime} \mathrm{d} t^{\prime}+\int_{0}^{t} \int_{E} f\left(\mathbf{x}^{\prime}, t^{\prime}\right) G \mathrm{~d} \mathbf{x}^{\prime} \mathrm{d} t^{\prime}$. 
This reduces the problem of estimating the STF $T(\mathbf{x}, t)$ to a straightforward computation of the quadratures, which can be done either analytically or numerically.

Setting $f \equiv 0$ in 11 and computing the remaining quadratures gives the natural soil temperature profile at large times (Appendix A),

$$
T_{\text {nat }}\left(x_{3}, t\right)=T_{m}-A \mathrm{e}^{-a x_{3}} \cos \left(\omega t-a x_{3}\right), \quad a=\sqrt{\frac{\omega}{2 \kappa}} .
$$

This solution is routinely used to represent the impact on thermal fluctuations at the ground surface on soil temperature [4, 20].

\subsection{Flat-panel GHE}

A temperature field induced by the flat-panel GHE with $f(\mathbf{x}, t)$ in 10$)$ is obtained from (11) as

$$
T(x, z, t)=T_{\text {nat }}(z, t)+\frac{1}{4 K} \sqrt{\frac{\kappa}{\pi}} \sum_{i=1}^{N} \mathcal{T}_{i}(x, z, t)
$$

where

$$
\mathcal{T}_{i}=\int_{t_{0}}^{t} \frac{q_{0}(\tau)}{\sqrt{t-\tau}} \exp \left[-\frac{\left(x-\xi_{i}\right)^{2}}{4 \kappa(t-\tau)}\right] \sum_{j=1}^{4} \nu_{j} \operatorname{erf}\left(\frac{\psi_{j}}{2 \sqrt{\kappa(t-\tau)}}\right) \mathrm{d} \tau
$$

and $\nu_{1}=\nu_{4}=1, \nu_{2}=\nu_{3}=-1, \psi_{1}=d+z, \psi_{2}=d-z, \psi_{3}=d+z+h$, $\psi_{4}=d-z+h$. Recall that to emphasize the two-dimensional nature of the $\mathrm{STF}$ the coordinates are relabeled as $x \equiv x_{1}$ and $z \equiv x_{3}$. If the GHE is operated by keeping the heat flux constant in any given day, i.e., by treating $q_{0}(t)$ is a step function at the daily scale, the contribution of the $i$ th panel is

$$
\mathcal{T}_{i}=\sum_{k=0}^{t-t_{0}-1} q_{0}\left(t_{0}+k\right) \int_{t-t_{0}-k-1}^{t-t_{0}-k} \exp \left[-\frac{\left(x-\xi_{i}\right)^{2}}{4 \kappa \tau}\right] \sum_{j=1}^{4} \nu_{j} \operatorname{erf}\left(\frac{\psi_{j}}{2 \sqrt{\kappa \tau}}\right) \frac{\mathrm{d} \tau}{\sqrt{\tau}}
$$

\section{Model Validation}

The experimental data collected at a field site of the Department of Architecture, University of Ferrara (Italy) are used to validate these analytical 
models. A detailed description of the GHE design and operations is provided in [23. Its brief summary is provided below. The laboratory tests identified the following soil properties: porosity 0.36 , bulk soil density $\rho=1720 \mathrm{~kg} / \mathrm{m}^{3}$, bulk specific heat $c=1950 \mathrm{~J} / \mathrm{kg} / \mathrm{K}$. The GHE consists of two (polypropylene) flat panels, each of which is $3 \mathrm{~m}$ long, $h=1 \mathrm{~m}$ wide, and $4 \mathrm{~mm}$ thick. The panels were placed $20 \mathrm{~mm}$ apart, forming a $6.02 \mathrm{~m}$ long GHE buried at $d=0.8 \mathrm{~m}$ below the ground. The panels were backfilled with sieved soil, and a dedicated drainage/irrigation system was laid over them to control soil moisture. Two digital probes measuring ground temperature, named v3 and v6 in Figure 2 , were placed at depths $z=2.03 \mathrm{~m}$ and $2.57 \mathrm{~m}$, respectively. Both the GHE size and the location of these sensors relative to the GHE are consistent with the two-dimensional solution (13).

A weather station at the field site recorded, among other atmospheric characteristics, the air temperature close to the ground surface and the soil temperature at depths $z=0.15,0.80,2.50,4.20 \mathrm{~m}$. Fitting the boundary condition (3) to the air temperature data from 2014 yields $A=10.8{ }^{\circ} \mathrm{C}$ and $T_{m}=16.3{ }^{\circ} \mathrm{C}$. Fitting (12) to the soil temperature data collected during two months in 2014 yields the value of thermal diffusivity $\kappa=4.41 \times 10^{-7} \mathrm{~m}^{2} / \mathrm{s}$. The predicted and observed natural soil temperature profiles are shown in Figure 3.
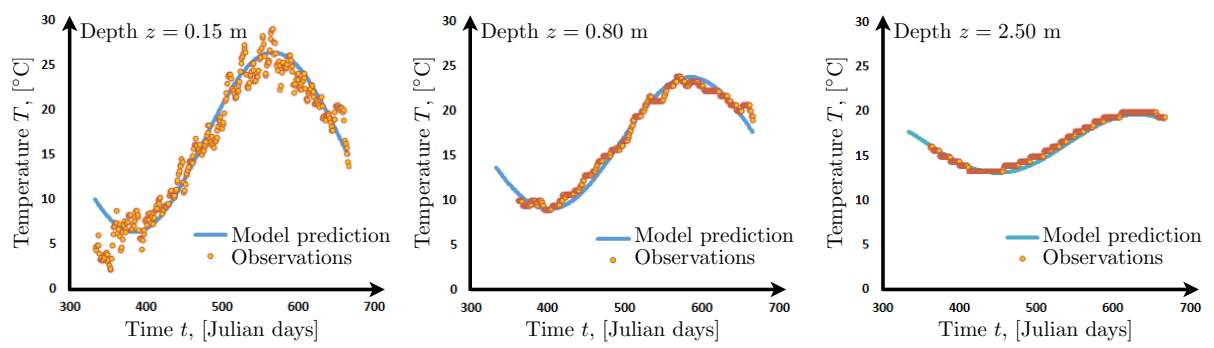

Figure 3: Predicted and observed natural (undisturbed) soil temperature $\left({ }^{\circ} \mathrm{C}\right)$. Measurements are collected by the surface monitoring stations at depths $z=0.15,0.80$ and $2.50 \mathrm{~m}$, from November 29, 2013 (333 Julian days) to October 28, 2014 (666 Julian days). The predictions are provided by equation 12 .

With the soil thermal properties thus determined, the solution 13 provides 
a fit-free prediction of the STF generated by the GHE. Figure 4 provides a comparison of this prediction with the soil temperature recorded by the sensors v3 and v6 from June 21, 2014 to September 8, 2014. During this time interval the flat panels were operating in the heating mode (i.e., heating the soil for cooling purposes) with the average heat flux $q_{0}=35 \mathrm{~W} / \mathrm{m}^{2}$. The points are clustered around the $45^{\circ}$ line, and their spread is virtually negligible. This demonstrates the accuracy of our modeling predictions, thus validating the analytical model 13 .
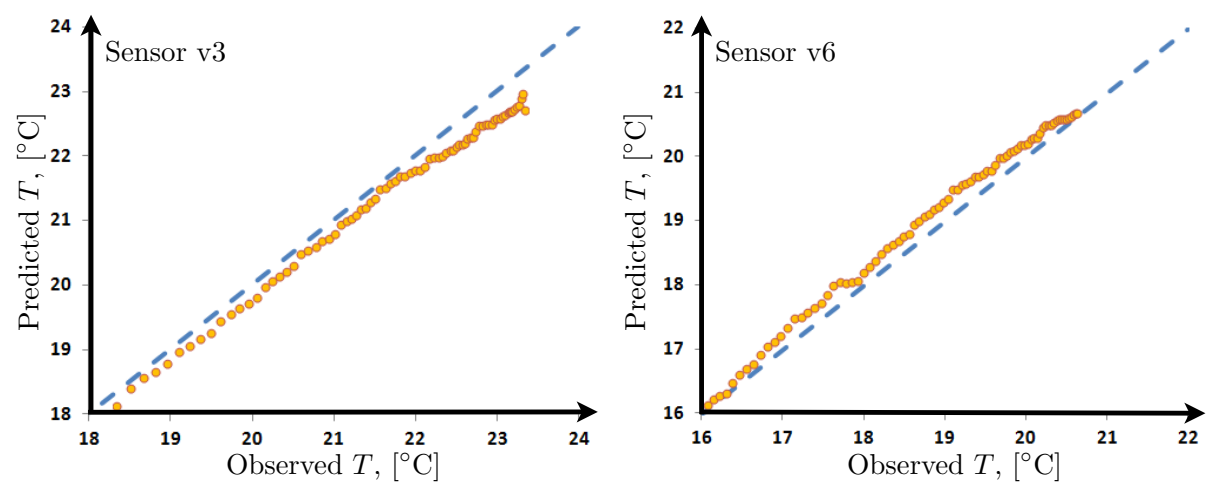

Figure 4: Comparison of the predicted and observed soil temperatures $\left({ }^{\circ} \mathrm{C}\right)$. The measurements are collected by the sensors v3 (first panel) and v6 (second panel), from June 21, 2014 (172 Julian days) to September 8, 2014 (251 Julian days). The predictions are given by equation 12 for the same time period.

\section{Model Forecasting}

We use the model to predict the dynamics of the STF induced by the GHE's operation during the cold season, i.e., within the time interval $\left[t_{0}=275\right.$ day, $t_{1}=$ 485 day] or October through April. The values of $q_{0}(t)$ for each day included in this time interval are deduced from the thermal energy needed to maintain a building's indoor temperature between $20^{\circ} \mathrm{C}$ and $25^{\circ} \mathrm{C}$. The energy requirement is related to the air temperature fluctuations, assumed to be equal to the temperature at the ground surface [see $[6$, for details]. Figure 5 a exhibits the hourly air temperature at the ground surface, its representation with the surface 
thermal wave (3), and the indoor building temperature. Figure $5 \mathrm{p}$ shows the

the corresponding heat flux $q_{0}$ estimated in [6].
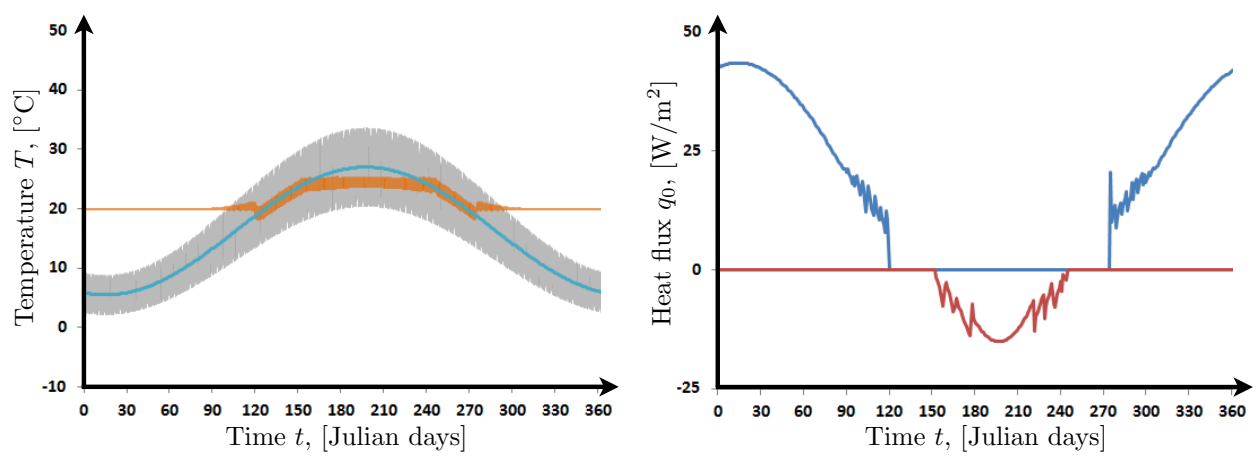

Figure 5: (a) Hourly temperature fluctuations at the ground surface, approximated surface thermal wave (as prescribed in [20]), and indoor building temperature for year 2014. (b) The corresponding heat flux $q_{0}(t)$ exchanged by the GHE and ambient soil during the same year.

These values of the heat flux $q_{0}$ are used in $(13)$ and $(14)$ to predict the $\mathrm{STF}$, whose temporal snapshots (at $t=405,485$ and 505 days) are displayed in Figure 6, together with their natural (undisturbed) counterparts. The minimum soil temperature, recorded at $t=405$ days, is $T \approx-0.5{ }^{\circ} \mathrm{C}$ near the GHE at depth $z=0.9 \mathrm{~m}$. This is due to the soil's delayed response response to the most critical ground temperature and to the consequent maximum energy exchanged by the GHE to reach the required indoor building temperature. At $t=405$ days, the GHE has the the largest effect on soil temperature $\left(T=-10{ }^{\circ} \mathrm{C}\right)$ at depth $z=1.1 \mathrm{~m}$; this effect decays rapidly with the distance from the GHE, becoming virtually negligible at depth $z=0.5 \mathrm{~m}$. In the end of the heating period $(t=485$ days $)$, the GHE influence diminishes, reaching a maximum of $T=-3.5^{\circ} \mathrm{C}$ at depth $z=1.2 \mathrm{~m}$. This diminished effect is to be expected, since the ground-surface temperature is higher and the energy requirement is smaller. The minimum temperature, $T \approx 10.5^{\circ} \mathrm{C}$, is predicted to occur at depth $z=1.4 \mathrm{~m}$, close to the GHE. The impact of the GHE decreases further at $t=505$ days, reaching a maximum of $T=-1.5^{\circ} \mathrm{C}$ at depth $z=2.3 \mathrm{~m}$ and becoming negligible away from the GHE. The minimum of the STF in this case, 
$T=13.0^{\circ} \mathrm{C}$, is reached at depth $z=2.7 \mathrm{~m}$.

\section{Summary}

\section{Acknowledgement}

This work was supported in part by Università di Bologna RFO (Ricerca Fondamentale Orientata) 2012 and 2013, by the Air Force Office of Scientific 

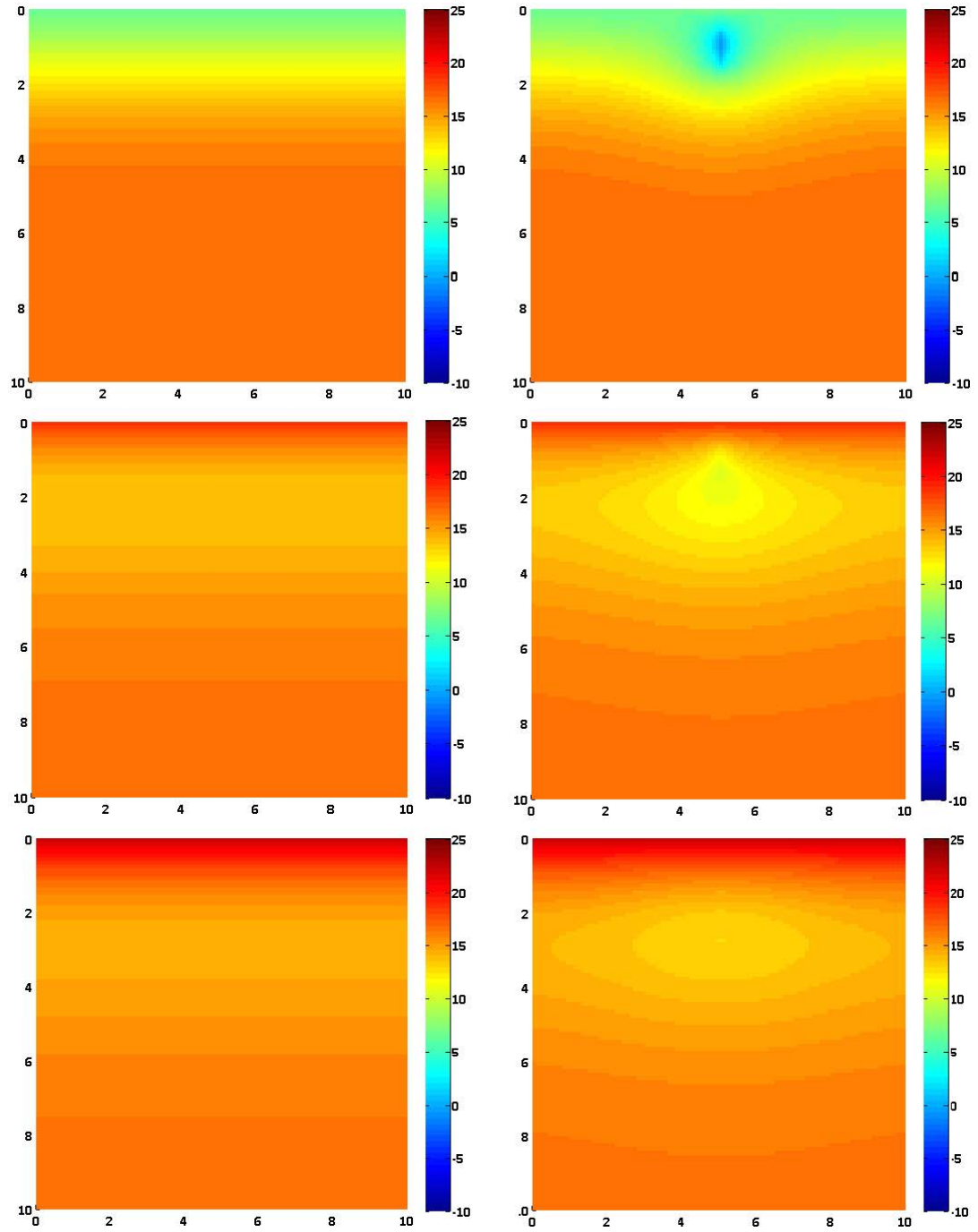

Figure 6: Temporal snapshots of natural (first column) and GHE-induced (second column) soil-temperature fields $\left({ }^{\circ} \mathrm{C}\right.$ ) at $t=405$ days (first row), 485 days (second row) and 505 days (third row). 
Research under Grant Number FA9550-12-1-12-1-0185, and by the National Science Foundation under Grant Number EAR-1246315.

\section{Appendix A. Derivation of Analytical Solutions}

The Green's function $G\left(\mathbf{x}, \mathbf{x}^{\prime}, t-t^{\prime}\right)$ satisfies

$$
\frac{\partial G}{\partial t^{\prime}}=\kappa \nabla^{2} G+\delta\left(\mathbf{x}-\mathbf{x}^{\prime}\right) \delta\left(t-t^{\prime}\right)
$$

subject to the homogeneous versions of the initial and boundary conditions 2 and (3). It is computed as the product of the corresponding one-dimensional Green's function,

$$
G\left(\mathbf{x}, \mathbf{x}^{\prime}, t-t^{\prime}\right)=\prod_{i=1}^{3} G_{x_{i}}\left(x_{i}, x_{i}^{\prime}, t-t^{\prime}\right)
$$

The one-dimensional Green's functions in the $x_{1}$ and $x_{2}$ directions are identical since they are defined on the infinite domain $x_{i} \in(-\infty,+\infty)$. They are given by [e.g., [24, page 353]

$$
G_{x_{i}}\left(x_{i}, x_{i}^{\prime}, t-\tau\right)=\frac{1}{2 \sqrt{\pi \kappa(t-\tau)}} \exp \left[-\frac{\left(x_{i}-x_{i}^{\prime}\right)^{2}}{4 \kappa(t-\tau)}\right], \quad i=1,2 .
$$

The one-dimensional Green's function in the $x_{3}$ direction is defined on the semiinfinite domain $x_{3} \in[0,+\infty)$. It is given by [e.g., 24, page 357]

$G_{x_{3}}\left(x_{3}, x_{3}^{\prime}, t-\tau\right)=\frac{1}{2 \sqrt{\pi \kappa(t-\tau)}}\left\{\exp \left[-\frac{\left(x_{3}-x_{3}^{\prime}\right)^{2}}{4 \kappa(t-\tau)}\right]-\exp \left[-\frac{\left(x_{3}+x_{3}^{\prime}\right)^{2}}{4 \kappa(t-\tau)}\right]\right\}$.

Substituting A.2 - A.4 into 11 with $f \equiv 0$ gives the natural temperature profile in the subsurface. The spatial integrals of the Green's function equal unity, so that 11 with $f \equiv 0$ gives rise to

$$
T(x, z, t)=T_{m}-\frac{A z}{2 \sqrt{\pi \kappa}} \int_{0}^{t} \frac{\cos \left(\omega t^{\prime}\right)}{\left(t-t^{\prime}\right)^{3 / 2}} \exp \left[-\frac{z^{2}}{4 \kappa\left(t-t^{\prime}\right)}\right] \mathrm{d} t^{\prime} .
$$

A change of integration variable, $\mu=z / \sqrt{\kappa\left(t-t^{\prime}\right)}$, yields

$$
T(\mathbf{x}, t)=T_{m}-A \mathrm{e}^{-a z} \cos (\omega t-a z)-\frac{A}{\sqrt{\pi}} \int_{0}^{z / \sqrt{\kappa t}} \cos \left(\omega t-2 \frac{a^{2} z^{2}}{\mu^{2}}\right) \mathrm{e}^{-\mu^{2} / 4} \mathrm{~d} \mu
$$


where $a=\sqrt{\omega /(2 \kappa)}$. The integral in A.6) represents the transition from the initial state. It tends to zero $t$ increases. This leads to 12 .

\section{References}

[1] G. Florides, S. Kalogirou, Ground heat exchangers - A review of systems, models and applications, Renew. Energ. 32 (15) (2007) 2461-2478.

[2] J. W. Lund, D. H. Freeston, T. L. Boyd, Direct application of geothermal energy: Worldwide review, Geothermics 34 (2005) 691-727.

[3] H. Yang, P. Cui, Z. Fang, Vertical-borehole ground-coupled heat pumps: A review of models and systems, Appl. Energ. 87 (2010) 16-27.

[4] S. P. Kavanaugh, K. Rafferty, Ground-source heat pumps design of geothermal systems for commercial and institutional buildings, in: Proceedings of ASHRAE 1997 Annual Conference, ASHRAE, Atlanta, GA, 1997.

[5] L. Rybach, W. J. Eugster, Sustainability aspects of geothermal heat pumps, in: Proceedings of the 27th Workshop on Geothermal Reservoir Engineering, Stanford, CA, 2002.

[6] M. Bottarelli, L. Gabrielli, Payback period for a ground source heat pump system, Int. J. Heat Technol. 29 (2011) 145-150.

[7] M. Bottarelli, V. Di Federico, Numerical comparison between two advanced HGHEs, Int. J. Low Carbon Tech. 7 (2012) 75-81.

[8] V. Ciriello, M. Bottarelli, V. Di Federico, Uncertainty-based analysis of variations in subsurface thermal field due to horizontal flat-panel heat exchangers, Procedia Environ. Sci. in press.

[9] H. Fujii, S. Yamasaki, T. Maehara, T. Ishikami, N. Chou, Numerical simulation and sensitivity study of double-layer Slinky-coil horizontal ground heat exchangers, Geothermics 47 (2013) 61-68. 
[10] D. Hillel, Fundamentals of soil physics, Academic Press, San Diego, CA., 1980.

[11] G. S. Campbell, J. D. Jungbauer Jr., W. R. Bidlake, R. D. Hungerford, Predicting the effect of temperature on soil thermal conductivity, Soil Sci. 158 (5) (1994) 307-313.

[12] A. Rabinovich, G. Dagan, T. Miloh, Underground heat conduction near a spherical inhomogeneity: theory and applications, Geophys. J. Int. 189 (3) (2012) 1521-1535.

[13] K. Roth, Soil Physics Lecture Notes, V2.2, Institute of Environmental Physics, Heidelberg University, Heidelberg, Germany, 2012.

[14] W. Sommer, J. Valstar, P. van Gaans, T. Grotenhuis, H. Rijnaarts, The impact of aquifer heterogeneity on the performance of aquifer thermal energy storage, Water Resour. Res. 49 (12) (2013) 8128-8138.

[15] R. B. Simms, S. R. Haslam, J. R. Craig, Impact of soil heterogeneity on the functioning of horizontal ground heat exchangers, Geothermics 50 (2014) $35-43$.

[16] D. A. de Vries, Heat transfer in soils, in: D. A. de Vries, N. H. Afgan (Eds.), Mass Transfer in the Biosphere, Part 1. Transfer Processes in the Plant Environment, John Wiley \& Sons, New York, 1975.

[17] T. R. H. Holmes, M. Owe, R. A. M. De Jeu, H. Kooi, Estimating the soil temperature profile from a single depth observation: A simple empirical heatflow solution, Water Resour. Res. 44 (2) (2008) doi:10.1029/2007WR005994.

[18] Z. H. Wang, Reconstruction of soil thermal field from a single depth measurement, J. Hydrol. 464-465 (2012) 541-549.

[19] D. M. Tartakovsky, S. P. Neuman, Transient effective hydraulic conductivities under slowly and rapidly varying mean gradients in bounded threedimensional random media, Water Resour. Res. 34 (1) (1998) 21-32. 
[20] UNI11466, Heat pump geothermal systems - Design and sizing requirements, Tech. rep., Italian Authority of Technical Regulation, Rome, Italy. (2012).

[21] D. M. Tartakovsky, Assessment and management of risk in subsurface hydrology: A review and perspective, Adv. Water Resour. 51 (2013) 247-260.

[22] D. M. Tartakovsky, S. P. Neuman, Transient flow in bounded randomly heterogeneous domains: 1. Exact conditional moment equations and recursive approximations, Water Resour. Res. 34 (1) (1998) 1-12.

[23] M. Bottarelli, A preliminary testing of a flat-panel ground heat exchanger, Int. J. Low Carbon Tech. 8 (2) (2013) 80-87.

[24] H. S. Carslaw, J. C. Jaeger, Heat conduction in solids, Clarendon Press, Oxford, UK, 1959. 